\title{
Association Between 16S RRNA Gene Sequencing of Gut Microbiota and Response to Antiviral Therapy in Chronic Hepatitis C Patients R.M.El-Badawy ${ }^{1}$, A.M.Matta ${ }^{2}$, T.E.El-Eraky ${ }^{1}$, A.M.Ahmed ${ }^{3}$ \\ ${ }^{1}$ Hepatology, Gastroenterology and Infectious Diseases Dept., Faculty of Medicine, Benha Univ., Benha, Egypt \\ ${ }^{2}$ Microbiology and Immunology Dept., Faculty of Medicine, Benha Univ., Benha, Egypt \\ ${ }^{3}$ Hepatology, Gastroenterology and Infectious Diseases Dept., Boulak El dakror general hospital, Giza, Egypt. E-Mail:dr.elshehry986@gmail.com
}

\begin{abstract}
Still there is percentage of HCV patients not responding to Direct Acting Antiviral Agents (DAAS), even the responder $\mathrm{HCV}$ patients are in need to follow up. Gut flora (Microbiota) include all the microorganisms in the intestine and liver can be greatly affected by changes in gut microbiota. The study was done to evaluate the association between gut flora and the response to DAAS in chronic HCV patients. Two groups; group 1 ( $\mathrm{No}=15$ of $\mathrm{HCV}$ responders patients) and group 2 (No=15 non responder HCV patients) treated by DAAS according to the treatment protocol of the Egyptian National Committee for Control of Viral Hepatitis (NCCVH). Healthy control subjects ( $\mathrm{No}=15)$ age and sex matched to the study groups as third group. Full history taking, clinical examination and all investigations were done plus stool culture and 16srRNA gene amplification and sequencing were done.The results show statistically significant difference between the patients (responders and non-responders) and control subjects .Microbiota which decreased in non-responder group than responder group (Lactobacillus brevis strain with $\mathrm{P}$ value: $0.013^{*}$, Pediococcus pentosaceus with $\mathrm{P}$ value: $0.014^{*}$, Clostridium tetani with $\mathrm{P}$ value: $0.006^{*}$, Shigella flexneri with $\mathrm{P}$ value: $<0.001^{*}$, Shigella dysenteriae $\mathrm{P}$ value: $0.008^{*}$, Shigella sonnei with $\mathrm{P}$ value: $0.026^{*}$ ). Microbiota which increased in non-responder group than responder group (Pseudomonas aeruginosa with $\mathrm{P}$ value: 0.003*, Streptococci with P value: 0.002*). Microbiota which increased in responder group than non-responder and control groups (Enterobacter hormaechei with $\mathrm{P}$ value: $0.013^{*}$ and Enterococcus fecalis with $\mathrm{P}$ value: $0.01 *$ ). In conclusion, the analysis of stool samples by using 16S rRNA gene of patients with chronic HCV infection (responders to DAAs and nonresponders) in comparison to healthy individuals is important issue. Patients with HCV had a few significant changes that may be related to liver-controlled homeostasis, protein synthesis, lipid digestion, or possibly to bacterial translocation, immune modulation, or a combination of all of the above mechanisms than healthy individuals. Even the responder patient needed to be followed up to modulates his microbiota changes to his health state. Non-responder group needs strict observation and modulation for their microbiota to be similar to the responder group and to avoid the development of HCC.
\end{abstract}

\section{Introduction}

Hepatitis C virus (HCV) infection represents an important global cause of chronic hepatitis, cirrhosis and HCC [1]. Chronic hepatitis $\mathrm{C}$ is prevalent in many countries as in Egypt (15\%), Pakistan (4.8\%) and China (3.2\%) [2]. The human gastrointestinal tract contains millions of microorganisms with up to 2000 different species of bacteria [3]. The gut flora begin to colonize shortly after birth and plays an important role in keeping the individual healthy by digestion improvement, vitamin production, bile acids generation, and modulation of immune mechanisms of the host [4]. Many factors, including diet, drugs, illness, stress, and lifestyle, affect the structure of community of gut microbiota and microflora, which has a rule in health [5]. Many studies showed a close relation between the gut microflora and disease development [6]. The use of 16S rRNA gene sequences to study bacterial phylogeny and taxonomy has been by far the most common housekeeping genetic marker used for a number of reasons. These reasons include (i) its presence in almost all bacteria, often existing as a multigene family, or operons; (ii) the function of the 16S rRNA gene over time has not changed, suggesting that random sequence changes are a more accurate measure of time (evolution); and (iii) the 16S rRNA gene (1,500 bp) is large enough for informatics purposes [7]. .In recent years, the relationship between the gut microbiota and the liver has been studied which is described as 'gut-liver axis' [8]. Many studies documented the involvement of intestinal microflora in alcoholic liver disease (ALD) and non-alcoholic steatohepatitis (NASH), liver cirrhosis and hepatocellular carcinoma (HCC) [9]. However, little is known about the relation between hepatic viruses and, especially hepatitis $\mathrm{C}$ virus infection and intestinal microflora. Here, we give an outline of the current knowledge on the association of the gut microbiota and the course of treatment in chronic hepatitis $\mathrm{C}$ patients.

\section{Aim of the work}

The aim of the present study is to evaluate the association between gut flora and the response of antiviral therapy for chronic HCV patients; responders, and nonresponders.

\section{Subjects and methods 3.1Subjects}

The prospective case study was conducted on 30 patients with chronic HCV patients from Benha University Hospitals and 15 healthy subjects served as a control group. All patients are adults aging between 18 and 65. All patients included provided a written consent before participation in treatment protocol of $\mathrm{HCV}$ and the 
protocol of this study approved by medical ethical committee of the Faculty of medicine, university of Benha and also approved by Central Administration of Research and Health development, Ministry of Health and Population. Subjects included in this study were classified into the following groups: Group I: This group included 15 patients with chronic hepatitis $\mathrm{C}$ who achieved sustained virological response (SVR).Group II: This group included 15 patients with chronic hepatitis $\mathrm{C}$ who didn't achieve SVR after 12weeks of DAAs therapy. Group III: This group included 15 apparently healthy subjects served as control group.

\subsection{Inclusion criteria}

1) Patients chronically infected with HCV confirmed with Anti-HCV positive and HCV RNA by positive PCR.

2) Patients $>18$ years old and $<65$ years old.

3) Patients with platelet count $>50,000 / \mathrm{cmm}$.

4) Patients with hemoglobin level $>10 \mathrm{~g} / \mathrm{dl}$.

5) Patients with INR $<1.7$

6) Patients with total bilirubin $<2 \mathrm{mg} / \mathrm{dl}$.

7) Treatment naïve or treatment experienced.

\subsection{Exclusion criteria}

1) Direct serum bilirubin $>2 \mathrm{mg} / \mathrm{dl}$.

2) Serum albumin $<2.8 \mathrm{~g} / \mathrm{dl}$.

3) International Normalization Ratio (INR) $\geq 1.7$

4) Inflammatory bowel syndrome.

5) Extra-hepatic malignancy except after two years of disease-free interval.

6) Pregnancy or inability to use effective contraception.

7) Patients with HBV co-infection.

8) Patients with history of Wilson disease or hemochromatosis.

9) Autoimmune liver disease

All the patients and controls were subjected to full history taking and complete clinical examination. All patients were considered eligible to participate in the current study. Patients received 12 weeks regimen of daily sofosbuvir $400 \mathrm{mg}$ with either daclatasvir $60 \mathrm{mg}$ (20 of 30) or ledipasvir $90 \mathrm{mg}$ (10 of 30). Some of patients received weight-based ribavirin (15 of 30). HCV-RNA PCR was done to assess treatment response at end of 12 weeks after completion of treatment. Treatment outcome was achieving a sustained virological response (SVR), which was defined as "response" and failure to achieve an SVR, which was defined as "non response".

- All laboratory investigations done according to the protocol for HCV patients as well as US and endoscopy.

\subsection{Routine laboratory investigations}

1- Complete blood picture.
2- Random blood sugar.

3- Kidney function tests including blood urea and serum creatinine.

4- Thyroid profile (TSH, free T3 and free T4).

5- Liver function tests, including serum bilirubin "total and direct", serum albumin and prothrombin time and INR.

6- Markers of liver injury: alanine transaminase (ALT), aspartate transaminase (AST) and alkaline phosphatase (ALP).

7- Serum $\alpha$-feto protein.

8- Viral markers including: hepatitis B surface antigen (HBsAg) and hepatitis $\mathrm{C}$ antibody (HCV-Ab) by ELISA.

9- HCV polymerase chain reaction (PCR) before treatment, after 12 weeks, 24 weeks from the end of treatment.

\subsection{Stool culture for Microbita Sampling \\ 1) Sample collection and DNA extraction}

Stool samples were collected in sterile containers from patients and healthy controls. Stool specimens were processed upon receipt in the microbiology laboratory on the date of collection.

\section{Organism identification \\ First day}

Routine bacterial stool cultures were inoculated onto various selective and differential media including MacConkey agar, blood agar and Sabouraud agar and incubated aerobically and anaerobically at $35^{\circ} \mathrm{C}$ according to standard laboratory methods.

Wet and Gram film was done to detect pus cells and bacteria then loopful from the sample was taken to do culture on different media as mentioned.

\section{Second day}

Culture media were examined for bacterial growth. Subcultures were done in cases of mixed growth.

\section{2) $16 \mathrm{~S}$ rDNA Identification}

Sequencing was performed at Clinilab laboratories in El-Maadi, Cairo, Egypt.

I- Isolate genomic DNA from samples.

II- Dilute genomic DNA for PCR.

III- Prepare the PCR reactions.

IV- Perform the amplification run.

V- (Optional) Analyze PCR products.

VI- Purify PCR products for cycle sequencing.

VII- Prepare cycle sequencing reactions.

VIII- Perform the cycle sequencing run.

IX- Purify extension products.

$\mathrm{X}$ - Configure the instrument for electrophoresis.

XI- Prepare samples and perform electrophoresis [10]. 


\section{Workflow}
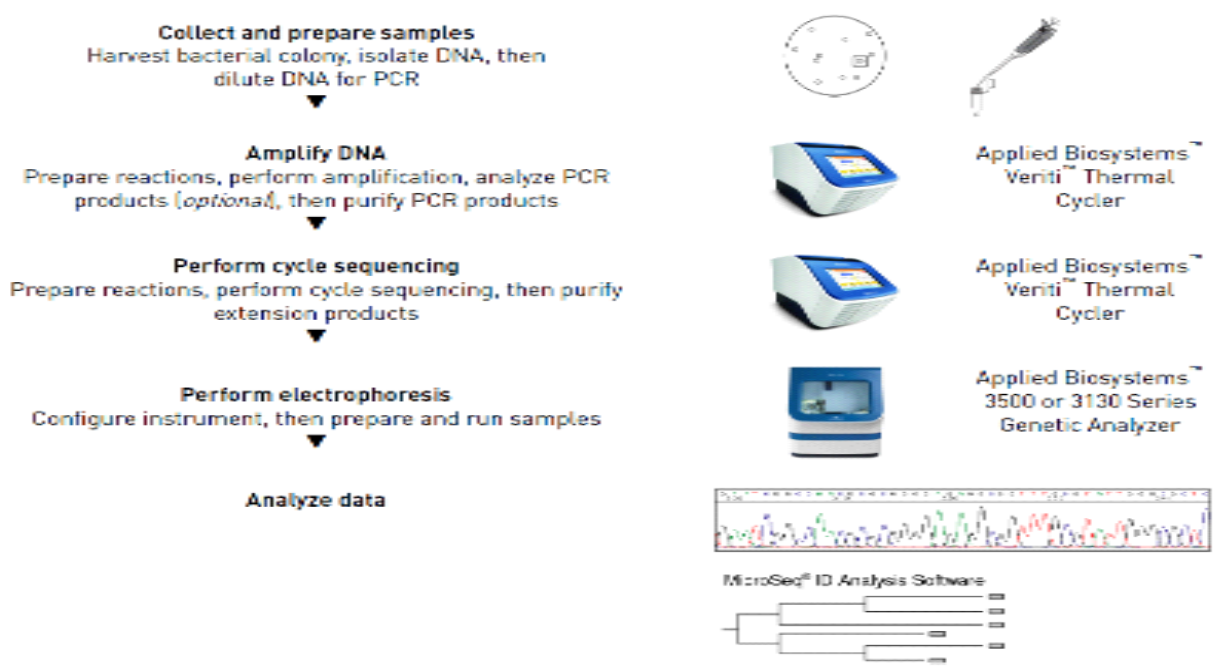

Fig (1) Results of 16s rRNA gene sequencing in all studied groups

\subsection{Statistical analysis Data management}

The clinical data were recorded in a report form. These data were tabulated and analyzed using the computer program SPSS (Statistical Package for Social Science) version 20 to obtain:

\section{Descriptive data}

Descriptive statistics were calculated for the data in the form of:

1) Mean standard deviation (+ SD) Median and interquartile range (IQR) for quantitative data.

2) Frequency and distribution for quantitative data.

\subsection{Analytical statistics}

In the statistical comparison between the different groups, the significance difference was tested using one of the following tests:

1) Student's t-test and Mann-Whitney test: Used to compare mean of two groups of quantitative data of parametric and non-parametric respectively.

2) ANOVA A test (F value) and Kruskal-Wallis test: Used to compare mean of more than two groups of quantitative data of parametric and non-parametric respectively.

3) Inter-group comparison of categorical data was performed by using Chi square (X2-value) and Fisher's exact test (FET).

$$
\begin{gathered}
x^{2}=\frac{\sum(\text { observed }- \text { expected })^{2}}{\text { Expected }} \\
\text { Expected }=\frac{\text { col.total } x \text { row total }}{\text { Grand total }}
\end{gathered}
$$

A $\mathrm{P}$ value $<0.05$ was considered statistically significant ${ }^{*}$ ) while $>0.05$ statistically insignificant. $\mathrm{P}$ value $<0.01$ was considered highly significant $(* *)$ in all analyses.

\section{Results}

Subjects included in this study were classified into the following groups: Group I: This group included 15 patients with chronic hepatitis $\mathrm{C}$ who achieved SVR.Group II: This group included 15 patients with chronic hepatitis $\mathrm{C}$ who didn't achieve SVR after 12 weeks of DAAs therapy. Group III: This group included 15 apparently healthy subjects served as control group.

The fecal microbiomes of HCV patients (responders and non-responders) and fifteen healthy individuals were sequenced by using 16s rRNA gene which revealed these results which discussed later.

Core bacterial taxa shared by each group (healthy controls and HCV patients) were identified. Overall, 22 distinct OTUs (operational taxonomic units) were conserved among all samples, constituting a core gut microbiome. This core set is characterized by genera Streptococcus, Ruminococcus, Clostridium, Faecalibacterium, Bacteroides, and Lachnospira in addition to some undefined members of families Enterobacteriaceae and Clostridiaceae. Most of these genera were differentially distributed among healthy and HCV patients.

\section{Group I (Responders)}

There was 2 microbiota increased and 2 microbiota decreased compared to control and non-responders.

\section{Group II (Non-Responders)}

There was increase for 2 microbiome in non-responders compared to the responders patients. There was a decrease 
for 6 microbiome in non-responders compared to the responders patients.

\section{Group III (control)}

There was increase for 4 microbiome in control compared to the responders patients.

There was increase for 6 microbiome in non-responders compared to the non-responder patients.

No statistical significant between the 3 groups with 8 microbiota.

We can summarize study results in 5 groups for Smooth and easy understanding

Group I : No statistical significant difference between Microbiota in all groups Table (1), fig (1).

1- Escherichia coli 5- Staph aureus

2- Proteus mirablis 6- Bacteroides fragilis

3-Klebsiela pneumonia 7- Clostridium perfringens

4- Enterobacter 8-Enterococcus faecium

Group II : Microbiota which decreased in non-responder group than responder group
1- Lactobacillus brevis strain P value: $0.013 *$

2- Pediococcus pentosace P value: $0.014^{*}$

3- Clostridium tetani P value: $0.006^{*}$

4 -Shigella flexneri P value $>: 0.001^{*}$

5 -Shigella dysenteriae P value: $0.008^{*}$

6- Shigella sonnei P value: $0.026^{*}$

Group III : Microbiota which increased in non-responder group than responder group

1- Pseudomonas aeruginosa P value: $0.003^{*}$

2-Streptococci P value: $0.002 *$

Group IV : Microbiota which increased in responder group than non-responder and control groups

1- Enterobacter hormaechei P value: $0.013 *$

2- Enterococcus fecalis P value: $0.01 *$

Group V : Microbiota which decreased in responder group than non-responder and control groups

1- Shigella boydii P value: $0.001^{*}$

2- Enterococcus durans P value: $0.002 *$

Table (1) Descriptive data of the demographic features and special habits in all studied groups.

\begin{tabular}{lccc}
\hline & $\begin{array}{c}\text { Responders } \\
\mathbf{N = 1 5}\end{array}$ & $\begin{array}{c}\text { Non responders } \\
(\mathbf{N = 1 5})\end{array}$ & Control (N=15) \\
\hline $\begin{array}{lcc}\text { Sex } \\
\text { Male }\end{array}$ & $11(73.3)$ & $8(53.3)$ & $10(66.7)$ \\
Female & $4(26.7)$ & $7(46.7)$ & $5(33.3)$ \\
Age & $56.67 \pm 5.82$ & $52.0 \pm 7.55$ & $36.27 \pm 14.01^{\mathrm{ab}}$ \\
Occupation & & & \\
Farmer & $3(20.0)$ & $1(6.7)$ & $1(6.7)$ \\
Non farmer & $12(80.0)$ & $14(93.3)$ & $14(93.3)$ \\
Residence & & & $7(46.7)$ \\
Rural & $9(60.0)$ & $8(53.3)$ & $8(53.3)$ \\
Urban & $6(40.0)$ & $7(46.7)$ & $10(66.7)$ \\
Marital status & & & $5(33.3)$ \\
Married & $11(73.3)$ & $13(86.7)$ & \\
Not married & $4(26.7)$ & $2(13.3)$ & $1(6.7)$ \\
Special habits & & & $14(93.3)$ \\
Smoker & $3(20.0)$ & $4(26.7)$ & \\
Non smoker & $12(80.0)$ & $11(73.3)$ & \\
\hline
\end{tabular}

Table (2) General and abdominal examination in responders and non-responders groups.

\begin{tabular}{lcccc}
\hline & $\begin{array}{c}\text { Responders } \\
\mathbf{N = 1 5}\end{array}$ & $\begin{array}{c}\text { Non responders } \\
(\mathbf{N = 1 5})\end{array}$ & Statistical test & $\begin{array}{c}\text { P } \\
\text { value }\end{array}$ \\
\hline $\begin{array}{l}\text { Jaundice } \\
\text { Pallor }\end{array}$ & $0(0.0)$ & $0(0.0)$ & $\wedge 0.21$ & 0.65 \\
$\begin{array}{l}\text { Lower } \\
\text { edema }\end{array}$ & $3(20.0)$ & $5(33.3)$ & $\wedge 0.17$ & 0.68 \\
$\begin{array}{l}\text { Contour } \\
\text { (Abdominal }\end{array}$ & $1(6.7)$ & $0(0.0)$ & $\wedge 0.96$ & 0.33 \\
$\begin{array}{l}\text { enlargement) } \\
\text { Liver size }\end{array}$ & $6(40.0)$ & $7(46.7)$ & $\mathrm{x}^{2}=0.14$ & 0.71 \\
$\begin{array}{l}\text { Enlarged } \\
\text { Average }\end{array}$ & & & & \\
& $1(6.7)$ & & & - \\
& $14(93.3)$ & $1(6.7)$ & &
\end{tabular}


Table (2) Continue

\begin{tabular}{|c|c|c|c|c|}
\hline \multicolumn{5}{|c|}{ Liver consistency } \\
\hline Firm & $15(100)$ & $15(100)$ & $\wedge 0.0$ & 1.0 \\
\hline Hard & $0(0.0)$ & $0(0.0)$ & & \\
\hline \multicolumn{5}{|l|}{ Spleen } \\
\hline Not felt & 13(86.7) & $9(60.0)$ & $\wedge 2.86$ & 0.22 \\
\hline Enlarged & $2(13.3)$ & $5(33.3)$ & & \\
\hline $\begin{array}{l}\text { Surgically } \\
\text { removed }\end{array}$ & $0(0.0)$ & $1(6.7)$ & & \\
\hline
\end{tabular}

Table (3) Complaint and Clinical history in responders and non responders groups.

\begin{tabular}{|c|c|c|c|c|}
\hline & $\begin{array}{l}\text { Responders } \\
\mathrm{N}=15\end{array}$ & $\begin{array}{c}\text { Non responders } \\
(N=15)\end{array}$ & Statistical test $\left(\mathrm{x}^{2}\right)$ & P value \\
\hline Abdominal pain & $9(60.0)$ & $5(33.3)$ & 2.14 & 0.14 \\
\hline \multicolumn{5}{|l|}{ Others } \\
\hline Bleeding per rectum & $2(13.3)$ & $0(0.0)$ & $\wedge 7.22$ & 0.09 \\
\hline Constipation & $0(0.0)$ & $1(6.7)$ & & \\
\hline Vomiting & $0(0.0)$ & $3(20.0)$ & & \\
\hline Chest pain & $0(0.0)$ & $1(6.7)$ & & \\
\hline Shortness of brith & $1(6.7)$ & $0(0.0)$ & & \\
\hline None & $12(80.0)$ & $10(66.7)$ & & \\
\hline Abdominal pain & $9(60.0)$ & $11(73.3)$ & 0.60 & 0.44 \\
\hline Fever & $6(40.0)$ & $1(6.7)$ & $\wedge 2.98$ & 0.08 \\
\hline Operations & $10(66.7)$ & $8(53.3)$ & 0.56 & 0.46 \\
\hline Diabetes mellitus & $6(40.0)$ & $4(26.7)$ & 0.60 & 0.44 \\
\hline Hypertension & $4(26.7)$ & $5(33.3)$ & $\wedge^{\wedge} 0.0$ & 1.0 \\
\hline Blood transfusion & $4(26.7)$ & $8(53.3)$ & 2.22 & 0.14 \\
\hline Bilharziasis & $6(40.0)$ & $6(40.0)$ & 0.0 & 1.0 \\
\hline $\begin{array}{l}\text { Anti } \\
\text { treatment }\end{array}$ & $10(66.7)$ & $14(93.3)$ & & \\
\hline No & $4(26.7)$ & $1(6.7)$ & $\wedge 3.23$ & 0.17 \\
\hline Oral & $1(6.7)$ & $0(0.0)$ & & \\
\hline Injection & & & & \\
\hline
\end{tabular}

Table (4) Hematologic parameters, renal functions and Liver profile in all studied groups.

\begin{tabular}{|c|c|c|c|c|c|}
\hline $\begin{array}{l}\text { Lab } \\
\text { investigation }\end{array}$ & $\begin{array}{l}\text { Responders } \\
\quad \mathrm{N}=15 \\
\text { Mean } \pm \text { SD }\end{array}$ & $\begin{array}{c}\text { Non responders } \\
(\mathrm{N}=15) \\
\text { Mean } \pm \text { SD }\end{array}$ & $\begin{array}{c}\text { Control }(\mathrm{N}=15) \\
\text { Mean } \pm \text { SD }\end{array}$ & $\begin{array}{l}\text { Statistic } \\
\text { al test } \\
\text { (F) }\end{array}$ & $P$ value \\
\hline HB gm/dl & $12.64 \pm 1.83$ & $13.33 \pm 1.54$ & $12.67 \pm 1.12$ & 0.97 & 0.39 \\
\hline $\begin{array}{l}\text { WBCs x } \\
1000 / \mathrm{cmm}\end{array}$ & $6.37 \pm 3.69$ & $6.34 \pm 1.74$ & $6.88 \pm 1.42$ & 0.22 & 0.80 \\
\hline $\begin{array}{l}\text { Platelets } \\
\text { x100/cmm }\end{array}$ & $210.6 \pm 83.02$ & $201.33 \pm 78.75$ & $317.67 \pm 60.51 \mathrm{ab}$ & 11.23 & $<0.001 * *$ \\
\hline Urea (mg/dl) & $38.53 \pm 8.42$ & $36.67 \pm 11.35$ & $31.27 \pm 5.81$ & 2.75 & 0.08 \\
\hline $\begin{array}{l}\text { Creatinine } \\
(\mathrm{mg} / \mathrm{dl})\end{array}$ & $1.08 \pm 0.34$ & $0.97 \pm 0.33$ & $0.96 \pm 0.19$ & 0.77 & 0.47 \\
\hline AST (IU/L) & $56.73 \pm 43.35$ & $58.27 \pm 30.61$ & $23.4 \pm 7.93 \mathrm{ab}$ & 6.07 & $0.005 * *$ \\
\hline ALT (IU/L) & $49.27 \pm 21.25$ & $58.67 \pm 32.2$ & $24.67 \pm 6.15 \mathrm{ab}$ & 9.09 & $0.001 * *$ \\
\hline $\begin{array}{l}\text { T.Bilirubin } \\
\text { Mean } \pm \text { SD } \\
\text { Median }(I Q R)\end{array}$ & $\begin{array}{c}1.09 \pm 0.58 \\
0.89(0.81-1.3)\end{array}$ & $\begin{array}{c}0.85 \pm 0.31 \\
0.8(0.7-1.0)\end{array}$ & $\begin{array}{c}0.95 \pm 0.22 \\
0.9(0.8-1.1)\end{array}$ & $\begin{array}{c}\mathrm{MW}= \\
1.3\end{array}$ & 0.52 \\
\hline $\begin{array}{l}\text { D. Bilirubin } \\
\text { Mean } \pm \text { SD }\end{array}$ & $\begin{array}{c}0.35 \pm 0.45 \\
0.2(0.15-0.30)\end{array}$ & $\begin{array}{c}0.19 \pm 0.09 \\
0.2(0.1-0.2)\end{array}$ & $\begin{array}{c}0.23 \pm 0.09 \\
0.2(0.2-0.3)\end{array}$ & $\begin{array}{c}\mathrm{MW}= \\
2.34\end{array}$ & 0.31 \\
\hline
\end{tabular}


Table (4) Continue

\begin{tabular}{lccccc}
\hline $\begin{array}{l}\text { Serum } \\
\text { albumin (g/dl) }\end{array}$ & $3.92 \pm 0.36$ & $3.98 \pm 0.41$ & $3.95 \pm 0.30$ & 0.11 & 0.90 \\
PT & $12.59 \pm 0.88$ & $13.53 \pm 1.92$ & $12.82 \pm 0.84$ & 2.09 & 0.14 \\
PC & $82.77 \pm 11.14$ & $79.4 \pm 13.44$ & $88.53 \pm 7.14$ & 2.70 & 0.08 \\
INR & $1.18 \pm 0.15$ & $1.26 \pm 0.23$ & $1.1 \pm 0.08 \mathrm{~b}$ & 3.22 & $0.05^{*}$ \\
\hline
\end{tabular}

Table (5) Liver profile and abdominal ultrasonographic findings in responders and non-responders groups.

\begin{tabular}{|c|c|c|c|c|}
\hline & $\begin{array}{c}\text { Responders } \\
\mathbf{N}=\mathbf{1 5} \\
\text { Mean } \pm \text { SD }\end{array}$ & $\begin{array}{c}\text { Non responders } \\
(\mathrm{N}=15) \\
\text { Mean } \pm \mathrm{SD}\end{array}$ & Statistical test & $P$ value \\
\hline AST (IU/L) & $56.73 \pm 43.35$ & $58.27 \pm 30.61$ & St $\mathrm{t}=0.11$ & 0.91 \\
\hline ALT (IU/L) & $49.27 \pm 21.25$ & $58.67 \pm 32.2$ & St $t=0.94$ & 0.35 \\
\hline Serum albumin (gm/l) & $3.92 \pm 0.36$ & $3.98 \pm 0.41$ & St $t=0.43$ & 0.67 \\
\hline PT & $12.59 \pm 0.88$ & $13.53 \pm 1.92$ & St $\mathrm{t}=1.72$ & 0.096 \\
\hline PC & $82.77 \pm 11.14$ & $79.4 \pm 13.44$ & St $t=0.75$ & 0.46 \\
\hline INR & $1.18 \pm 0.15$ & $1.26 \pm 0.23$ & St $\mathrm{t}=0.99$ & 0.33 \\
\hline \multicolumn{5}{|l|}{ Liver size } \\
\hline Enlarged & $6(40.0)$ & $6(40.0)$ & - & - \\
\hline Average & $9(60.0)$ & $6(60.0)$ & & \\
\hline Shrunken & $0(0.0)$ & $0(0.0)$ & & \\
\hline \multicolumn{5}{|l|}{ Liver texture } \\
\hline Normal & $15(100)$ & $15(100)$ & - & - \\
\hline Coarse & $0(0.0)$ & $0(0.0)$ & & \\
\hline \multicolumn{5}{|l|}{ Cirrhotic } \\
\hline Homomgenity & $15(100)$ & $15(100)$ & - & - \\
\hline \multicolumn{5}{|l|}{ Homogenous } \\
\hline $\mathrm{PV}$ diameter $/ \mathrm{cm}$ & $\begin{array}{c}0.89 \pm 0.19 \\
0.9(0.7-1.1)\end{array}$ & $\begin{array}{c}0.98 \pm 0.23 \\
0.9(0.8-1.2)\end{array}$ & $Z=1.03$ & 0.30 \\
\hline Pv patency & $15(100)$ & $15(100)$ & - & - \\
\hline Patent & & & & \\
\hline Spleen size & & & $\wedge 1.29$ & 0.71 \\
\hline Normal & $10(66.7)$ & $8(53.3)$ & & \\
\hline Enlarged & $5(33.3)$ & $6(40.0)$ & & \\
\hline Surgically removed & $0(0.0)$ & $1(6.7)$ & & \\
\hline Spleen collaterals & $0(0.0)$ & $2(13.3)$ & $\wedge 0.54$ & 0.48 \\
\hline
\end{tabular}

Table (6) Results of 16s rRNA gene sequencing in all studied groups.

\begin{tabular}{|c|c|c|c|c|c|}
\hline Organism & $\begin{array}{c}\text { Control } \\
(\mathrm{N}=15)\end{array}$ & $\begin{array}{c}\text { Responders } \\
(\mathbf{N}=15)\end{array}$ & $\begin{array}{c}\text { Non responder } \\
(\mathbf{N}=15)\end{array}$ & $\begin{array}{c}\text { Statistical } \\
\text { test }\end{array}$ & $P$ value \\
\hline Lactobacillus brevis strain & $11 / 15(73.3 \%)$ & $8 / 15(53.3 \%)$ & $3 / 15(20 \%)$ & 8.72 & $0.013 *$ \\
\hline Pediococcus pentosaceus & $12 / 15(80 \%)$ & $8 / 15(53.3 \%)$ & $4 / 15(26.6 \%)$ & 8.57 & $0.014 *$ \\
\hline Escherichia coli & $15 / 15(100 \%)$ & $15 / 15(100 \%)$ & $15 / 15(100 \%)$ & - & - \\
\hline Proteus mirablis & $6 / 15(40 \%)$ & $11 / 15(73.3 \%)$ & $11 / 15(73.3 \%)$ & 4.73 & 0.094 \\
\hline Klebsiela pneumoniae & $5 / 15(33.3 \%)$ & $9 / 15(60 \%)$ & $10 / 15(66.6 \%)$ & 3.75 & 0.15 \\
\hline Enterobacter & $6 / 15(40 \%)$ & $8 / 15(53.3 \%)$ & $11 / 15(73.3 \%)$ & 3.42 & 0.18 \\
\hline Enterobacter hormaechei & $5 / 15(33.3 \%)$ & $12 / 15(80 \%)$ & $5 / 15(33.3 \%)$ & 8.72 & $0.013 *$ \\
\hline Pseudomonas aeruginosa & $2 / 15(13.3 \%)$ & $2 / 15(13.3 \%)$ & $10 / 15(66.6 \%)$ & 12.13 & $0.003 * *$ \\
\hline Staph aureus & $5 / 15(33.3 \%)$ & $6 / 15(40 \%)$ & $7 / 15(46.6 \%)$ & 0.56 & 0.76 \\
\hline Streptococci & $3 / 15(20 \%)$ & $5 / 15(33.3 \%)$ & $12 / 15(80 \%)$ & 12.06 & $0.002 * *$ \\
\hline Bacteroides fragilis & $15 / 15(100 \%)$ & $14 / 15(93.3 \%)$ & $11 / 15(73.3 \%)$ & 4.81 & 0.11 \\
\hline Clostridium tetani & $13 / 15(86.6 \%)$ & $6 / 15(40 \%)$ & $5 / 15(33.3 \%)$ & 10.18 & $0.006 * *$ \\
\hline Clostridium perfringens & $10 / 15(66.6 \%)$ & $7 / 15(46.6 \%)$ & $5 / 15(33.3 \%)$ & 3.38 & 0.19 \\
\hline Shigella flexneri & $15 / 15(100 \%)$ & $10 / 15(66.6 \%)$ & $4 / 15(26.6 \%)$ & 17.65 & $<0.001 * *$ \\
\hline
\end{tabular}


Table (6) Continue

\begin{tabular}{llllll}
\hline Shigella dysenteriae & $15 / 15(100 \%)$ & $10 / 15(66.6 \%)$ & $8 / 15(53.3 \%)$ & 9.88 & $0.008^{* *}$ \\
Shigella sonnei & $7 / 15(46.6 \%)$ & $13 / 15(86.6 \%)$ & $6 / 15(40 \%)$ & 7.99 & $0.026^{*}$ \\
Shigella boydii & $15 / 15(100 \%)$ & $5 / 15(33.3 \%)$ & $15 / 15(100 \%)$ & 23.27 & $<0.001^{* *}$ \\
Enterococcus fecalis & $15 / 15(100 \%)$ & $14 / 15(93.3 \%)$ & $9 / 15(60 \%)$ & 8.88 & $0.01^{*}$ \\
Enterococcus durans & $9 / 15(60 \%)$ & $6 / 15(40 \%)$ & $15 / 15(100 \%)$ & 12.6 & $0.002^{* *}$ \\
Enterococcus faecium & $8 / 15(53.3 \%)$ & $7 / 15(46.6 \%)$ & $13 / 15(86.6 \%)$ & 5.86 & 0.053 \\
\hline
\end{tabular}

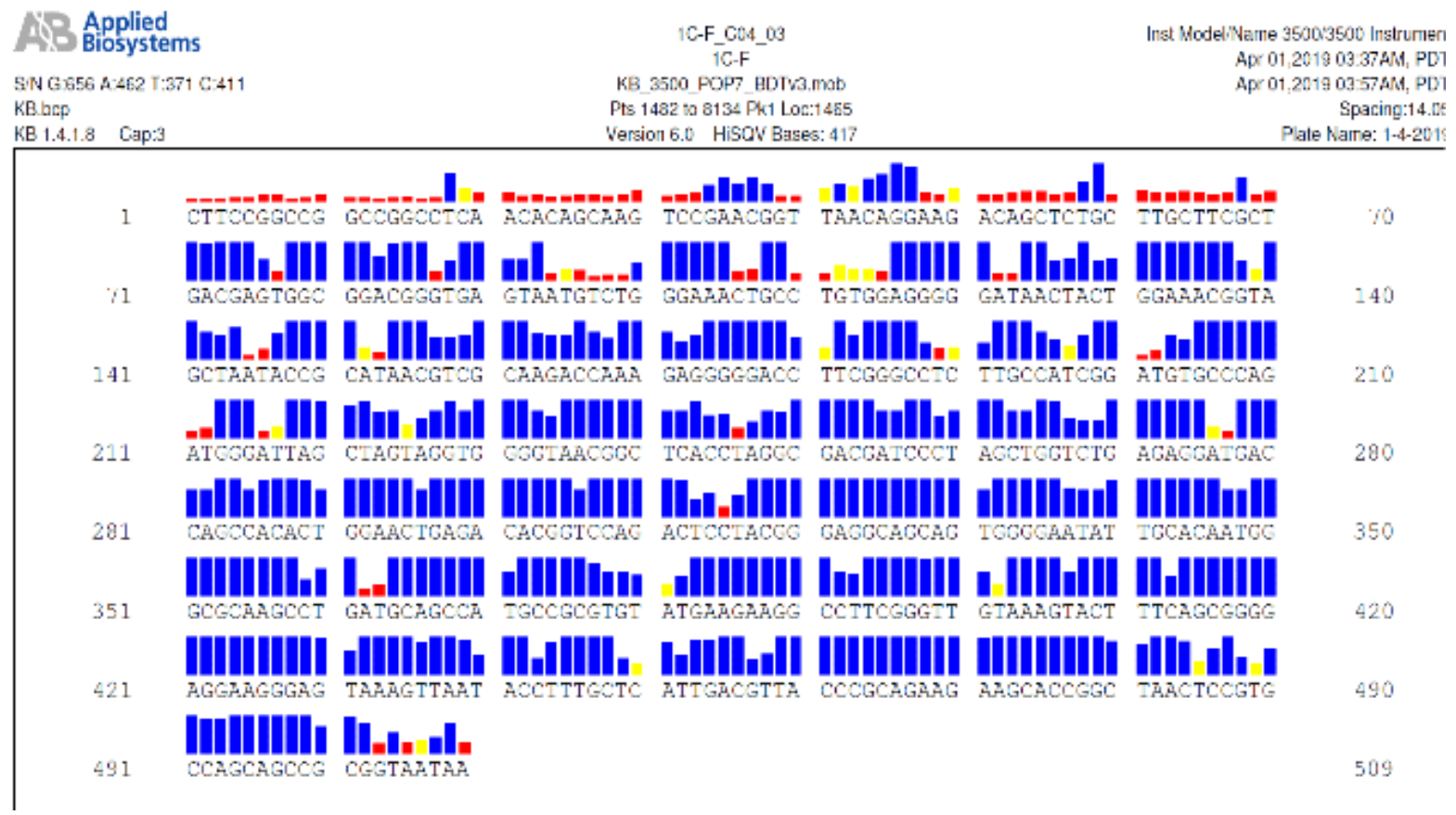

Fig (1) Results of 16s rRNA gene sequencing in control group.

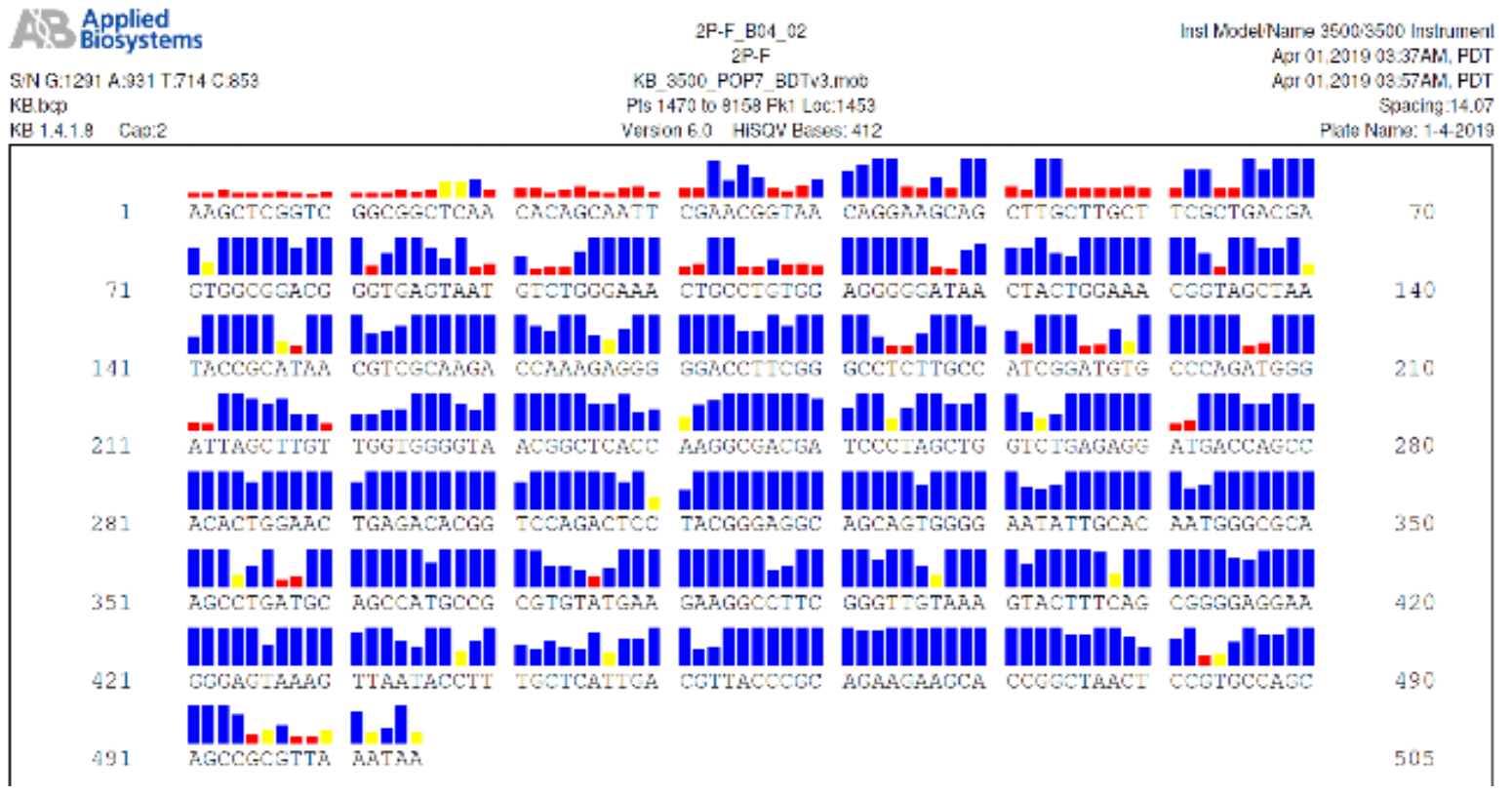

Fig (2) Results of 16s rRNA gene sequencing in Responder group. 


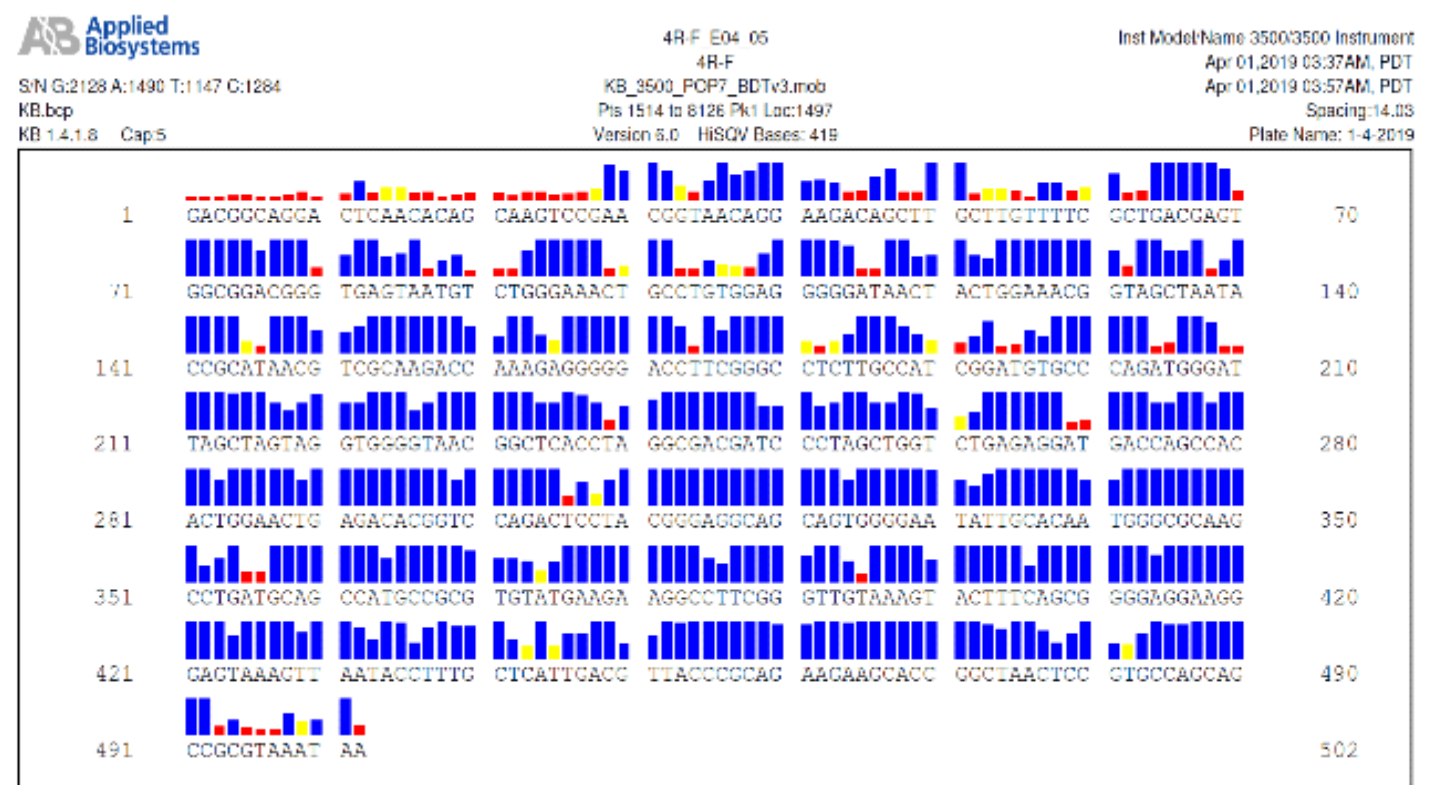

Fig (3) Results of 16s rRNA gene sequencing in non-responder group.

\section{Discussion}

Hepatitis $\mathrm{C}$ virus (CHC) is a major health concern worldwide and although often clinically asymptomatic, $\mathrm{HCV}$ infection is histologically an insidiously progressive disease leading to liver fibrosis, cirrhosis and HCC [11].

Approximately, 130-150 million people are infected with HCV; each year, besides an estimated 399000 people die from HCV related complications including fatty liver (cirrhosis), malignant neoplastic disease (hepatocellular carcinoma) and liver failure. Unluckily, many people with HCV only know about their infection when they experience symptoms of cirrhosis or HCC [12].

Egypt has the highest prevalence of HCV worldwide $(15 \%)$ and the highest frequency of HCV-4 responsible for almost $90 \%$ of infections and a major cause of chronic hepatitis, liver cirrhosis, hepatocellular carcinoma and transplantation in the country. Although HCV-4 is the cause of nearly $20 \%$ of the HCV infection worldwide [13].

Egypt reports the highest prevalence of $\mathrm{HCV}$ worldwide, ranging from $6 \%$ to more than $40 \%$ among different regions and demographic groups [14].with average 15\% [15].In 2017 was reported to be about $6 \%$ [16].

Since the emergence of HCV infection, it has always been a public health problem in Egypt. It took over the liver disease burden after schistosomiasis. HCV infection has a unique situation in Egypt early from its history and will continue till elimination, hopefully, in the near future [17].

Emerging data suggest a strict interaction between the gut microbiota, health, and disease [18].

The usefulness of 16S rRNA gene sequencing as a tool in microbial identification is dependent upon two key elements, deposition of complete unambiguous nucleotide sequences into public or private databases and applying the correct "label" to each sequence. Years ago the overall quality of nucleotide sequences deposited in public databases was questionable, since many depositions were of poor quality [19].

The use of 16S rRNA gene sequencing in the clinical laboratory is becoming commonplace for identifying biochemically unidentified bacteria or for providing reference identifications for unusual strains. 16S rRNA gene sequencing for definitive microbial identifications and for publication requires a harmonious set of guidelines for interpretation of sequence data that needs to be implemented so that results from one study can be accurately compared to another [20].

The gut microbiota composition of the hepatic patients is affected by diet, nutritional status, alcohol intake, impaired metabolism of bile acids, altered gastrointestinal motility and use of antibiotics [21].

However, little is known about the interaction between hepatotropic viruses particularly the $\mathrm{HCV}$ infection and human gut microbiota. Here, we give an overview of the current understanding on the association between the intestinal microflora and host reaction in the course of treatment of chronic hepatitis $\mathrm{C}$ and describe the diversity of gut microbiota in responders and non-responders and comparing them also with healthy individuals.

The aim of the study was to evaluate the association between 16srRNA gene and its impact on response to antiviral therapy in chronic HCV Patients with DAAS.

Our study was conducted at the outpatient clinic of Hepatology, Gastroenterology and Infectious Diseases Department at Benha University Hospital and Benha Fever 
Hospital in the period from January 2018 to January 2019 on 15 patients with chronic hepatitis $\mathrm{C}$ who respond to treatment (Group I), 15 patients with chronic hepatitis $\mathrm{C}$ who failed to respond to treatment (Group II) in addition to 15 apparently healthy control subjects (Group III).

In this study, there was no statistically significant difference between the responders and non-responders groups as regarding age with a mean age 52 years.

Similar results was detected by Paul and his colleagues (2016) who reported the same results and concluded that SVR not affected by age of patients but it gives more success rate when age of patients less than 50 years [23], in addition to Asselah and colleagues (2017) who documented that no statistically significant difference between the two groups as regarding age with a mean age 56 years [24], on the other hand Hezode and his partners (2017) found that age older than 40 years is an independent predictor of a reduced SVR [25].

In this study there was non-statistical significant difference between the groups as regards sex, occupation, residence and special habits.

In this study, there was no significant difference as regards ALT, AST, serum albumin and INR level between responders and non-responders. This finding is in agreement with the finding of Sato and colleagues (2017) who stated that no significant difference in ALT, AST and INR level between responders and non-responders [26]. Similar results were detected by Asselah and colleagues (2017) who said that baseline alanine aminotransferase (ALT) levels were not associated with treatment response [24].

There are no significant differences between responders and non-responders groups as regards complaint as abdominal pain, bleeding per rectum, constipation, vomiting, chest pain, shortness of breath and other symptoms ( $\mathrm{p}>0.05)$.

There are no significant differences between responders and non-responders groups regarding history as abdominal pain, fever, operations, DM, hypertension, blood transfusion, and Bilharziasis ( $>>0.05)$.

There are no significant differences between responders and non-responders groups regarding clinical manifestations $(p>0.05)$.

There are no significant differences between the responders and non-responders groups regarding liver size, texture and homogenicity as well as portal vein patency, splenic size and collaterals detected by abdominal ultrasonography ( $\mathrm{p}>0.05)$.

The study revealed that there is no difference between all studied groups as regards Bacteroides fragilis being present in the stool samples of $100 \%$ of all subjects. This finding was in agreement with Patricia and colleagues (2019) who reported $100 \%$ incidence in both responders and non-responders [27].
An Egyptian study on chronic HCV patients, compared with the healthy controls, have shown at the phylum level an abundance of Bacteroidetes in the HCV patients [28].

We observed a higher abundance of Enterobacter in $\mathrm{HCV}$-infected patients. This result was in accordance with that of Bajaj (2016) who showed a peculiar gut microbiota composition in the chronic hepatitis $\mathrm{C}$ patients when compared with the healthy subjects including main families of Enterobacteriaceae, Clostridiales, and Lachnospiraceae [29].

Concerning Clostridiales, our study showed that Clostridium perfringens were more in healthy subjects .In this regard, Aly and partners (2016) stated that the healthy subjects have shown the abundance of Clostridium genus [28].

\section{Conclusion}

In conclusion, the analysis of stool samples by using 16S rRNA gene of patients with chronic HCV infection (responders to DAAs and non-responders) in comparison to healthy individuals is important issue. Patients with HCV had a few significant changes that may be related to liver-controlled homeostasis, protein synthesis, lipid digestion, or possibly to bacterial translocation, immune modulation, or a combination of all of the above mechanisms than healthy individuals.

Even the responder patient needed to be followed up to modulates his microbiota changes to his health state.

Non-responder group needs strict observation and modulation for their microbiota to be similar to the responder group and to avoid the development of HCC.

\section{Acknowledgment}

We would like to thank all participants of this study. We would also like to thank Prof. Reda Mohammed Salem El-Badawy, Prof. Amal Mounir Matta, Dr. Tamer ElEraky El Azab and Dr.Rasha Abdel-Hameed El-Sayed Ali, and all the Physicians from the Hepatology, Gastroenterology and Infectious Diseases Department at Benha University Hospital for their help in patient's recruitment, collection of demographic data from the patients included in the study and bioinformatics analyses.

\section{References}

[1] Keith R. Neal, S .Ramsay, BJ. Thomson, WL. Irving, Excess mortality rates in a cohort of patients infected with the hepatitis $\mathrm{C}$ virus: a prospective study. Gut, Vol. 56, pp.1098-104, 2007.

[2] World health organization. Http://www.who.int/mediacentre/factsheets/fs 164/en/i ndex.html Hepatitis C, Fact sheet $\mathrm{N}^{\circ} 164$, July 2013.

[3] M. Minemura, Y.Shimizu, Gut microbiota and liver diseases. World J Gastroenterol, Vol.21, PP.1691$1702,2015$.

[4] M. Wan, H.El-Nezami ,Targeting gut microbiota in hepatocellular carcinoma: probiotics as a novel 
therapy. Hepatobiliary Surg Nutr, Vol.7, PP.11$20,2018$.

[5] J. Henao-Mejia, E .Elinav, C. Jin, L. Hao, WZ. Mehal, $\mathrm{T}$.Strowig, Inflammasome mediated dysbiosis regulates progression of NAFLD and obesity. Nature, Vol.482, PP.179-185,2012.

[6] L .Biedermann, G. Rogler, The intestinal microbiota: its role in healthand disease. Eur J Pediatr, Vol.174(2), PP.151-167,2015.

[7] J. B. Patel, 16S rRNA gene sequencing for bacterial pathogen identification in the clinical laboratory. Mol. Diagn;Vol.6, PP.313-321,2001.

[8] H. Fukui, Gut microbiota and host reaction in liver diseases. Microorganisms, Vol.3 (4), pp.759791,2015 .

[9] L. Abenavoli, E .Scarpellini, S. Rouabhia, C .Balsano, F.Luzza, Probiotics in non-alcoholic fatty liver disease: which and when. Ann Hepatol, Vol.12(3), PP.357-363,2013.

[10] MicroSEQ ${ }^{\mathrm{TM}} 500$ 16S rDNA Identification Quick Reference.

[11] AM. Attallah, GE .Shiha, MM. Omran, KR. Zalata , A discriminant score based on four routine laboratory blood tests for accurate diagnosis of severe fibrosis and/or liver cirrhosis in Egyptian patients with chronic hepatitis C. 2006 Mar;vol.34(3), PP.163-9. Epub 2006 Feb 14, 2006.

[12]WHO, Global report on access to hepatitis $\mathrm{C}$ treatment, 2017.

[13] Egyptian Journal of Medical Human Genetics, vol. 16, (4), pp. 291-298,2015.

[14]EM. Lehman , ML. Wilson , Epidemic hepatitis C virus infection in Egypt: estimates of past incidence and future morbidity and mortality. J Viral Hepat, Vol.16(9), PP.650-8,2009.

[15] World health organization , Monitoring and evaluation for viral hepatitis $\mathrm{B}$ and $\mathrm{C}$ : recommended indicators and framework http://www.who.int/medi/acentrefactsheets/fs164/en/i ndex.html,2013.

[16] National treatment programme of hepatitis C in Egypt, Hepatitis C virus model of care. J Viral Hepat, Vol.24(4), PP.262-267,2017.

[17] Ahmed Elgharably, Asmaa I Gomaa, Mary ME Crossey, J. Peter Norsworthy, Imam Waked,

[18]D .Simon Taylor-Robinson, Hepatitis C in Egypt past, present, and future. Int J Gen Med, Vol.10, pp.16,2017 .
[19] L .Biedermann, G. Rogler, The intestinal microbiota: its role in health and disease. Eur J Pediatr, Vol.174 (2), pp.151-67, 2015.

[20]C. A. Petti, Detection and identification of microorganisms by gene amplification and sequencing. Clin. Infect. Dis, Vol.44, PP.1108$1114,2007$.

[21] M. J. M. Boudewijns, P. D. J. Bakkers Sturm, W. J. G. Melchers, 16S rRNA gene sequencing and the routine clinical microbiology laboratory: a perfect marriage? J. Clin. Microbiol,Vol.44, PP.3469-3470,2006.

[22] M.Minemura, Y. Shimizu, Gut microbiota and liver diseases. World J Gastroenterol, Vol.21, PP.1691$1702,2015$.

[23]C .Paul, T .Asselah, K .Reddy, N. Mobashery, R .Redman, RA .Vilchez, Ombitasvir plus paritaprevir plus ritonavir with or without ribavirin in treatmentnaïve and treatment- experienced patients with genotype 4 chronic hepatitis virus infection. Lancet, Vol.385, PP.2502- 09,2016.

[24] T .Asselah, KR. Reddy, T .Hassanein, M .Berenguer, K. Fleischer-Stepniewska, P .Marcellin, Ombitasvir plus paritaprevir plus ritonavir with or without ribavirin in treatment-naive and treatment-experienced patients with genotype 4 chronic hepatitis $\mathrm{C}$ virus infection. Lancet, Vol.(15), PP.605-608,2017.

[25]C.Hézode, B. Roula, M.ElKhashab, Ombitasvir, paritaprevir, and ritonavir plus ribavirin in adults with hepatitis $\mathrm{C}$ virus genotype 4 and cirrhosis. Lancet, Vol.(16), PP.30001-2,2017.

[26] K.Sato, Y.Yamazaki, T.Ohyama, Combination therapy with daclatasvir and asunaprevir for dialysis patients infected with hepatitis $\mathrm{C}$ virus.World J. Clin. Cases, vol.16, PP.88-93,2017.

[27] P.M.Patricia, I.María, J.María, Short-term effects of direct-acting antiviral agents on inflammation and gut microbiota in hepatitis $\mathrm{C}$-infected patients.European Journal of Internal Medicine,2019.

[28] A.R. Aly, A. Abdel Reheem, A.O. El Gendy, Gut microbiome alterations in patients with stage 4 hepatitis C, doi, Vol.10.1186, PP.s13099-016-01242,2016.

[29] J.S. Bajaj, Potential mechanisms of action of rifaximin in the management of hepatic encephalopathy and other complications of cirrhosis. Aliment Pharmacol Ther, Vol.43 (Suppl 1), PP.11-26,2016. 